\title{
Michael Kotulla Verfassungsrechtliche Aspekte im Zusammenhang mit der Einführung eines Umweltgrundrechtes in das Grundgesetz
}

Die Norwendigkeit zur Einführung eines Umweltgrundrecbtes wird kontrovers diskutiert. Die Gegner balten eine menschenwürdige. Umwelt rechelich niche für durchsetzbar. Die Befürworter, zu denen der Autor gebört, meinen, daß cin Umwelegrundrecht in das bestehende System der durch das Grundgesetz verbürgten Schutzgïter $z$ integrieren ist. Dies würde zu einer spürbaren Siärkung des rechtlichen Umweltschutzes fübren.

Die Red.

\section{Aksgangslage}

Umwcleschucz wird heuce zweifelsfrei als erstrangiges staatliches und geselischaftliches Anlicgen mir Verfassungsrang begriffen. Materiell-rcchclich dokumentierı dies in besonderer Weise die Verankerung des Schutzes der natürlichen Lebensgrundlagen durch den im Grundgeserz' (GG) als Staatsziel formulierten Ar. 20a. „Der Staac 4 - so heißc es dort - "schürzt auch in Verantwortung für die künfrigen Generacionen durch Legislativc, Exekurive und Judikative die natürlichen Lebensgrundlagen im Rahmen der verfassungsmäßigen Ordnung. « Dem Staat ist damit - ähnlich wie bereics in Arr. 1 so Abs. 1 der Wcimarer Reichsverfassung ${ }^{2}$ - eine ausdrückliche Verpflichrung zum Schutz der Umwelt auferlegt, freilich ohne daß der Bürger daraus cigenc subjekrive Rechre grgen oder auf hoheirliches Handeln herzuleiten vermag.'

\section{Feblen eines Umweltgrundrechtes im $G G$}

Derartige subjekrive Rechie auf den Schutz der natürlichen Lebensgrunḋlagen können sich auf der Ebene des GG allein aus den Gnodrechren ergeben. Nur sie gewahrleisten dem Einzeinen als Mensch und/oder als Bürger gegenüber dem Staac cinforderbare Fundamentalrechte. ${ }^{*}$ Doch sucht man nach cinem expressis verbis in der Bundesverfassung verankerten, den Schurz der natürlichen Lebensgrundlagen oder den Schutz einer menschenwürdigen Uinwelt statuierenden sog. „Umwelt-

1 Vom 23. 5.1949 (BGBI. I S. I), zuletzt geandert durch Gesetz zul' Änderung des Grundgestizes vom 26.) 1998 (BGBl. I S.610).

2 Verfassung des Deursclien Reiches vom 11.8.1919 (RGBl., S. 1383)-Text von An. 150 Abs. I WRV

"Die Denkmaler der Kunst, der Geschiche und der Natur sowie die Landschaft genießen den Schurz und dic Pflcge des Staztcs.

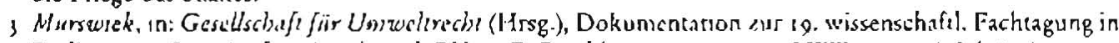
Burlin, ig95, $\mathrm{S}_{40}$ (44 f., 79): vgl, auch BVerwG, Beschl. vom 13-4. 1995, NJW 1995, 2648 (2649).

4 Hesse, Grandzige des Verfassungsrechts, 20. Aufl, t995. Rdл. 283. 
grundrechr " ebenso vergeblich wie nach einem sich möglicherweisc aus einer $\mathrm{Zu}$ sammenschau der Inhalte des bestehenden Grundrechtekaraloges der Verfassung herleitbaren, spezifisch ökologisch geprägten Grundrecht. Jedenfalls räumen die durch das GG gewährieistetcn Grundrechce nach mitcherweile nahezu einhelliger Auffassung kcinc für den Umweltschurz bedeutsame Grundrechtsposition ein, deren subjekciv-rcchelicher Schutz über die in den Art. Iff. GG genannten Rechisgüter (Leben, Gesundheit, Freiheit und Eigentum) hinausginge.' Insbesondere der im Schrifttum ${ }^{6}$ unternommene Versuch, aus Art. 2 Abs. 2 S. I GG (Recht auf Leben, körperliche Unversehrcheir) i.V.m. Art. x Abs. I GG (Rechr auf ein menschenwürdiges Leben) ein allgemeines Grundrecht auf eine menschenwürdige Umwelt herzuleiten, stieß zu Recht auf breite Ablehnung.? Für ein Grundrecht diesen Zuschnitts geben weder der Wortlaut noch die Entstehungsgeschichte der zu diesen Zweck bemühten Verfassungsvorschriften Anhalrspunktc. ${ }^{8}$ Das gilt erst recht für die Annahme eines sozialen Grundrechres auf Umweleschutz, welches vom Staat Leistungen vor allem in Form faktischer oder rechricher Zuwendungen verlangr. ${ }^{9}$ Dieser Befund findet für die Verfassungsebene der Länder seine Entsprechung: Zwar enthalten einige Landesverfassungen durchaus als Umweltgrundrechre zu qualifizierende Vorschrifcen. ${ }^{\circ}$ Doch gehen diese keinesfalls über den herkömmlichen Individualschucz von Leben, Gesundheit, Freiheit und Eigentum vor Beeinträchtigungen hinaus, ndie aus Veränderungen der natürlichen Lebensgrundlagen cntstehen " Gleiches hat umso mehr noch für die sonstigen Grundrechrsbestimmungen in den Landesverfassungen ohne besonderen ökologischen Zuschnitr zu gelten. Somit müssen de constiturione laca alle sich nicht unmitrclbar lebens-, gesundheits- oder eigentumsgefährdend auswirkenden Schädigungen der Umwelt von jedernaann hirgenommen werden.

\section{Stärkung der Umweltschutzbelange}

Demgegenüber garantiertc ein grundgesctzlich positiviertes Umweltgrundrecht scinem Inhaber - unter allerdings noch zu konkrerisierenden Vorausseczungen - ein subjektives Rechr auf den Schutz der für ihn wescntlichen ökologischen Verhälonisse durch die staacliche Gewalt. Damit wäre unter Berufung auf das Umweltgrundrecht für den Einzelnen staåliche Tätigkeir wie Untätigkeit auch jenseits der unmittelbaren Eigenbecroffenheit von Leben, Gesundheit und Eigentum angreifbar. Das härte nichr nur gravierende Auswirkungen im Falle verfassungsrechtlicher Streitigkeiten vor dem Bundesverfassungsgeriche (BVerfG), sondern wegen der mit dem Umweltgrundreche begründeren persönlichen Klagebefugnis auch für den Verwaltungsgerichtsprozeß." Die insoweit das Prozeßrecht beherrschende, der Vermeidung von Popularklagen und dadurch cinem dem Einzelnen garantierten effektiven gerichtli-

\footnotetext{
; BVerwG, Un. vom 29.7.1977, E s, 211 (219) - Ausführlich zu diesem Thomenkreis erwa Bindr, UPR $1982,241(242 f)$

6 Liscke, DOV 1976, 289 ff.; Rlspp, JZ 1971, 401 (403); Sening, BayVBl. 1976, 72 (76): W. Weber. DVBI. 1971. $80 \mathrm{sff}$.

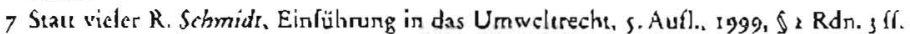

8 In dicsem Sime auch Siober, Handbuch des Wirschafisverwalnungs- und Umwelerechis, 1989, S. 429.

9 Karpen, in: Thitme (Hrsg), Umweleschutz im Recht, 1988, S.9 (17).

10 Vgl. Art. 14 i Abs.; S. I BayVert, 39 Abs. 2 BbgVerf, 12 Abs. 2 MVVerf.

II So An. 39 Abs. 2 BbgVerf. - die wohl z.Z. am weitesten gehende Umweltgrundrechtsbestimmung in ciner Landesverfassung. Die Art. ${ }_{4}$ I Abs. 3 S. I BayVerf und 12 Abs. 2 MVVerf gewährleisten bloßß ein Reche auf Erholung in der freien Natur bzw. auf freien Zugang zur Landschaft.

12 Vgl. hierzu auch Brönneke, ZUR 1993, Is3 (1 54 f.).
} 
chen Rechisschurz dienende "Schutznormcheorie «äßı nämlich grundsäızlich nur Klagen bei Betroffenheit eigener Rechtspositionen zu. ${ }^{3}$ Dementsprechend verlangt auch die von $\$ 42$ Abs. 2 V $\mathrm{wG} G$ als Zulässigkeitsvoraussetzung für einc Klage geforderte Klagebefugnis zumindest die Geltondmachung ciner möglicherweise dem Kläger selbst zustehenden Rechrsposition. Ein nach Maßgabe des Art. $4_{4}^{2}$ GG aut Landesverfassungsebene statuierbares Umweltyrundrccht stellte zu einem bundesverfassungsrechtlich verankerten indes keine gleichwertige Alternative dar. Es crreichte nicht einmal annähernd die Wirkkraft eines im GG vcrankerten Umweltgrundrechtes. Abgeschen von der terricorialen Beschränktheir gewährleistete ein solches Grundrecht wegen Art. 3 I GG keinen Schucz gegenüber nach Bundesrceht zu vollzichenden hoheitlichen Maßnahmen. ${ }^{4}$ Zwar wäre die durch das Umweltgrundrechc bewirkce Ausweitung der Eigenbetroffenheit bundesrechulich auch auf einfachgesezzlichem Wege möglich. Doch bliebe damir gegenüber der Grundrechtslösung im GG der erhebliche Nachreil verbunden, daß dieses Recht u.U. mit einlachen parkamentarischen Mehrheiten wieder beseitigt werden könnce.

Desweiteren stünde zu erwarcen, daß die Verankerung eines Umweltgrundrechtes im GG in der Bevölkerung cin verstärktes Bcwußtsein für die in ihrer Umgebung licgenden Umwelressourcen erzeugte. In gewisser Weise würde dadurch die bislang eher anonyme, nicht selten überforderce staarliche Umweltschurzüberwachung von einer solchen flankiert, die diejenigen betrieben, die es anginge."s

Auch könnte ein Umweltgrundrecht eine * Kernbestandsgarantie für das gektende Umwcltrecht ${ }^{16}$ implizieren. Damit wäre namentlich den rechtserzenden Gewalten die Unterschreiong bestimmter rechrlicher Mindeststandards zum Schutz der narürlichen Lebensgrundlagen unstatthaft. Elementare Regelungsbcreicle unterlägen einerscirs einem legislativen Verschlechtenngsverbot, andererseirs würden im Falle gravierender Regelungsdefizite Nachbesserungen einforderbar. Diesc könnten zwar nicht soweil gehen, vom Geserzgeber den Erlaß einer den eigenen Vorstellungen entsprechenden konkreten Maßnahme zu verlangen. Die Auswahl des Mittels muß allein der Legislative uberlassen bleiben. Doch härte die Gesetzgebung dafür zu sorgen, daß das grundrechtich geforderte Umweltschutzniveau durch ihre Rechtsctzungsarbeit crreicht wird.

Schließlich zöge ein solches Grundrecht einc beschleunigte und vertiefte Prioritätenverschiebung zugunsten des Umweltschutzes nach sich." Das wiederum zwänge insbesondere den Gesetzgeber, die Umwelrschurzgeserzgebung im Lichce des neuen Grundrechtes zu beurteilen und (noch) stärker an den Bedürfnissen des Umivelrschutzes auszurichten.

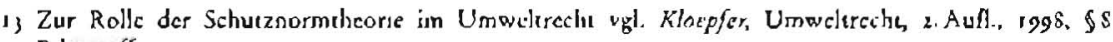
Rdin. 17 ff.

14 Durch Art, 142 GG werden Grundrechtsbestimmungen der Länder nicht zu höherrangigem Recht, das über sonstigem Bundesrecht stande, sondern bleiber Landesreche mit Nachrang gegenüber Bundesrecht

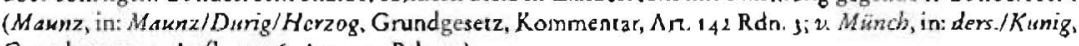
Grundgesetz, 3. Aufl., 1996, An. 142 Rdn. 2.).

1s Dieses Grundrecht stünde zudem in tiner logischen Bezichung zur parallelen Einfuhrung ciner enuprechenden Grundpflicht. - Zur Diskussion um die verfassungsrecheliclie Verankenung einer Umwelegrundpflicht vgl. Fïbr, NuR 1998, 6 il.

16 So Bock, Univcleschutz in Spiegel von Verfassungsrecht und Verfassungspolirik, 1990, S. 2 z.

17 Dazu auch Seciger, Mensch und Unweli, 1975, S. 66, 73 ff. und Maus, JA 1979, $2 \$ 7$ If. 

rechies

Im Zuge der zunehmenden allgemeinen Sensibilisierung für die Norwendigkeir verstärktcr Umwelrschurzanstrengungen ist die Einführung cines speziellen Umweltgrundrechres zum Schutz einer menschenwürdigen Umwelı bzw. der nacürlichen Lebensgrundlagen des Menschen in das GG seit den frühen siebziger Jahren von Politik und Rechtswissenschaft kontrovers erörtert ${ }^{18}$, namenclich von letzterer aber überwiegend mit folgenden Argumenten abgelehnt worden:"

- Menschenwürdige Umwelı wie mnaürliche Lebensgrundiagen ließen sich als Schutzgut nicht hinlänglich individualisiercn, um verfassungsunmitrelbar Ziellkonflikıc mit anderen (gleichrangigen) Schutzgütern besteben zu können. Dies gelce gerade für die Schutzgüter Luft und Wasser, Landschaft und Artenvielfalc. Ferner fehle cinem darauf bezogenen Grundrecht der Charakıer eines gegen den Staat gerichteten Abwehrrechrs, wodurch das Verständnis der gegenwärtigen Grundrechte relariviert würde. Der cinem Umwelcgrundrecht im wesentichen anhaftende Zuschnitt eines auf staatliche Leistung gcrichteren sozialen Tcilhabercchtes führe überdies zu der nicht justitiabel beantworbaren Frage, worin die vom Staat konkret geschuldete Leistung bestehen solle. Kurzum - man sieht durch die Einführung eines Umrveltgrundrechts eine "Verunsicherung des Verfassungsrechts « voraus ${ }^{20}$, die eine Glaubwürdigkeicskrise für das GG heraufbeschwören könne. Als Folge dieser primär verfassungssystemarischen Bcdenken sei überdies eine die Verwaltungsgerichrsbarkeir überfordernde Prozeßlawine zu befürchten.

Nicht zuleczt angesichts dieser sters von Ncuem angeführten, bislang nur unzurcichend auf ibre Stichhaltigkeit überprüften Ablehnungsgründe wird die weitere Diskussion um die Einführung eines Umweltgrundrechtes in das $G G$ als crledigr angesehen. Diese - wie zu zeigen sein wird - etwas voreilige Sichtweise versperre den Blick auf die mit cinem derartigen Grundrecht verbundenen Chancen für den Schutz der natürlichen Lebensgrundlagen.

\section{Verfassungssystematischer Rabmen für die Einfübrung eines Umwelt- grundrechts in das Grundgesetz}

Ungeachcer des zusäczlichen Nutzens, der von einem im GG verankerten Umweltgrundrecht für den Umweltschutz zu erwarten wäre (oben I. 2.), ist die beharrlich angezweifelte veriassungssystemarische Kompatibilirät eines solchen Grundrechts (oben I. 3.) der Hauprgrund, der dessen Einführung entgegenstehen soll. Den Kern-

$18 \mathrm{Vgl}$. Steilungnahme des Rates van Sichzierstandigen für Umweltfragen im Umweltgutachten 1974,

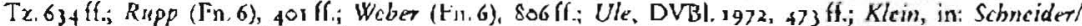
Golz (Hrsg.), Festschr. f. W. Weber (1974), S. 643 ff.: Rauschning, in: Schneider/Götz (Hrsg.), Festschr. 1. W. Weber (1974), S. 719 (f.; Bothe/Klem, ZaoRV i4 (197i), 3s iff.; Stciger (Fn. 17), 1975; Dellmann, DOV 1975, s88ff,; Külz, DVBI. 197s, i8gff.; Lucke (Fn.6), 289ff; Klocoler, Zum Grundrecht auf Unwclischutz, 1978; Sening. BayVBI. 1978.205f,; Maus (Fr. 17), 28, ff, H. Huber, in: Umwcll und

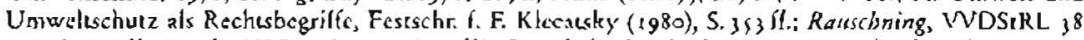
(1980), 18 ff., Benda, UPR 19\$2, 291 ( 242 ff.); Pernsisaler, in: Rack (Hrsg.), Gundrechurclorm, 198s,

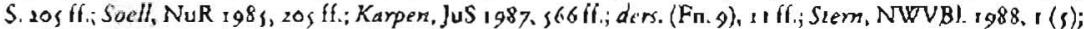
Bock (Fn. 16), S. 233 ff; Bronncke (Fn. 12), 153 ff.

19 Vgl. erwa Klein (Fn. 18), S. $6_{43}$ fl.; Dullmann (Fn. 18), 588 ff.; Klocefer (Fn. 18), S. j8 i. (allerdings vor dic Wahl gestellt: Umweltgrundrecht oder Staatszielbestimmung, ncindeutig amwelegrundrecht); Rapsehning (Fn. 18), 178f; Socll (Fn. 18), insbes. 209-2r1 ; Rupp (Fn. 6), fol If: Karpen (Fn. 9), S. gff.; Scholx, in: Masnz/Durng/Herzog, Grundgeseiz, Kommentar (32. Licf. 1996), Arr. $20 x$ Rdn. 12.

20 Klein (Fn. 18), S. 661. 
punkt der nachfolgend anzustellenden Überlegungen bildet deshalb der von der Grundrechtskonzeption des GG abgesteckte Rahmen, in den sich jedes neu dort aufzunehmende Grundrecht einzupassen härte. Diesen zugrundelegend wird geprüft, ob ein Umweltgrundrecht - entgegen den stereoryp angeführten Behauptungen der hierzu herrschenden Ansicht - in das bestehende Gesamrsystem der grundrechelich durch das GG verbürgten Schutzgücer integrierbar ist." "Erweist sich dies als möglich, spräche nicht nur aus Sicht eines effektiveren Schutzes der natürlichen Lcbensgrundlagen vieles dafür, die Aufnahme eines Umweltgrundrechis in das GG doch noch anzustreben.

\section{Die dem Grundgeset: zugrundeliegende Grundrechtskonzeption}

Ganz im Sinne der klassischen liberalen Grundrecheskonzeption ${ }^{22}$ dienen die Grundrechce im GG prinzipiell der Abwehr staatlicher Maßnahmen." Um die Grundrechce zu optimaler rechelicher Wirksamkeit zu bringen, sind sic in letzter Konsequenz zu einklagbaren subjekriven Rechten ausgebildet. "Die Verfassungsrechtsprechung und die Staatsrechtslehre haben den Grundrechten zwar eine Vielzahl weiterer Funktionen zugewiesen. Hervorzuheben sind insbesondere ibr Schurzpflichten, bisweilen leistungs- bzw. reilhaberechelicher oder Einrichungsgarantien vermittelnder Cliarakter. Dennoch gebührt der Abwehrfunkrion im deurschen Verfassungsrecht eindeutig der Vorrang. Deswegen müBte ein im GG verankertes Umweltgrundrecht in erster Linic als Abwehrrechc konzipierbar sein (dazu unter 2.). Alle sonstigen Funkcionen eines Grundrechtes treren lediglich ergänzend binzu, ohne die Abwchrfunkrion modifizieren, verdrängen oder sogar ersetzen zu können." Sic bleiben jedoch ungeachtet dessen dem Grundrechtssystem des GG verrrauce Schutzreflexe, die den insoweir zur Verfügung stehenden Beurteilungsrahmen der nachfolgend für das Umweltgrundrecht auszulocenden Systcmkompatibilität erweitern. Aus diesem Grund wird zudem auf die Rolle dieser Funklionen im Hinblick auf das hier in Rede slehende Grundrecht eingegangen (dazu unter 3.-s.).

\section{Umweltgrundrecht als Abwehrreche}

Abwehrechte schützen Berciche der privaten Selbstbestimmung des Einzelnen und schirmen sic ab gegen das Eindringen der öffentlichen Gewalt. ${ }^{26}$ Sic weisen trotz ihrer unterschicdlichen sprachlichen Fassung hinsichtlich der sachlichen Gewährleistung im wesentlichen einheitliche formale Struksurcn auf. Diese sind denkbar cinfach zugesclinitten auf ein einziges Gegenüber: auf den staatlichen Eingriff, der - beträfe er das Umweltgrundrecht - entsprechend zu steuem, zu kanalisieren und zu mäßigen wäre.

Die Abwehrrechte lassen sich auf wenige gemeinsame Grundelemente zurückführen,

$\therefore$ Vgl. zu dicscr Vorausscizung Scholy (Fn. 19), An. 201 GG, Rdn. 13.

21 Dizu cuva Schlink. EuGRZ 1984. 417 ff.

2) Vgl. z.B. dic sundige Rechisprecluung des BVerfG, insbesondere Urteil vom 15. 1. 1958, E 7, 198 (204): Beschl. vom 31, 10.19\$4, E 68, 193 (205).

24 Isenser, in: ders./Kirchbof, Handbuch des Staztsreclics, Bd, V. 1992, S111 Rdn. 22.

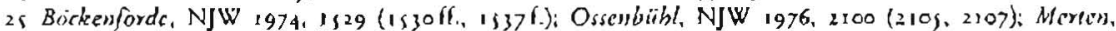
VorwArch 73 (1982), 103 ff.; /sensec (Fn. 24), \$11 Rdn. 11 m.w.N

26 Iscnser (Fn. 24). $\{111$ Rdn. 2. 
die zwingend auch auf cin in das GG einzuführendes Umweltgrundrecht übertragbar

scin müßten: Das sind die mit dem Grundrecht angesprochenen Subjekte (a.), dessen Schurzbereich ${ }^{27}$ (b.), der Eingriff in den Schurzbereich (c.) sowic die mögliche Rechiferigung des Eingriffs (d.).

\section{a. Grundrechtssubjekte}

Prinzipiell kann jede staatsangehörige oder ausländische natürliche oder inländische juristische Person, dic der deurschen Staarsgewalc in ihren unterschiedlichen Erscheinungen unterworfen ist, grundrechrsberechtigt sein. Dies gilt auch im Hinblick auf den Schutz der nazurlichen Lebensgrundlagen. Diesen indes - ganz im Sinne ökozentrisch ausgerichteter Schuzzvorstellungen - eigene Gundrechtspositionen zuzubilligen, liefe den Vorstellungen rom Grundrecht als einem personenabhängigen Abwehrrecht zuwider. Grundrechtsverpflichtet ist prinzipiell jeder - hier der auf die Nacurressourcen einwirkende - Hoheitsträger.

\section{b. Scbutzbereich}

Der sachliche Inhalt und Umfang des Grundrcchtsschutzes ergibe sich aus dem Schutzbereich. Er bezcichnec den verfassungsrechticls ausgegrenzten Sektor der Lebenswirklichkeit, der dem staatlichen Eingriff verschlossen oder jedenfalls nur nacla bestimmten verfassungsrechrlichen Kautelen zugänglich ist. ${ }^{28}$ In ihm sind das Schurzgur und die porentielle Reichweitc des Grundrechrsschutzes umschrieben. Das Schutzgut wäre in unserem Falle die »Umwelt * bzw. die »natürlichen Lebensgrundlagena des Menschen. Beide Bezeichnungen lassen sich synonym verwenden und vereinen in sich die gesamte nacürliche, die Basis des menschlichen Lebens bildende Umgebung, auclı wenn anthropogene Einwirkungen sie mitclerweile erheblich verändert haben. Erfaßt werden die Umweltmedien Luft, Wasser und Boden sowie Pflanzen, Tiere und Mikroorganismen in ihren Lebensräumen, einschließlich der Wechselwirkungen. ${ }^{29}$ Diese Grundlagen müssen im konkreren Fall für ein physiologisch gesundes menschliches Dasein notwendig sein. Das impliziert auch das von einer unbecinträchtigren Nacurlandschaft vermittelte positive Lebensgefühl des Menschen $^{30}$, welches durch negative landschafesästhecische Einflüsse (z.B. Verunstaltungen von bislang unberührten Naturräumen) mitunter empfindlich gestört werden kann.

Hinter der potentiellen Reichweite des Grundrechisschurzes verbirgt sich das Ausmaß der zu schützenden, d. h. zu bewahrenden und gegebenenfalls wiederherzustellenden, nacürlichen Lebensgrundlagen des Einzelnen. Für die Bestimmung des Ausmaßes ist selbstverständlich die unverzichrbare Inanspruchnahme der ökologischen Ressourcen durch den Menschen in der Industriegesellschaft zugrundezulegen. Dieser (ge)braucht bseine u Unwelt als Potential für Produktion, Konsum und Regeneration sowie als Mittel zur Aufnahme von Abprodukten aller Art. Ohne Umweltbelastungen in einem gewissen Umfange ist die Bewalurung der bestehenden Gesellschafisscruktur und der mic ihr verbundenen Lebensformen letztlich genauso wenig mojglich, wie umgekelon die Erhaltung der Gebrauchsfähigkeic der Umwelt für alle Menschen (über)lebenswichtig bleibr. So gesehen verfolgen Beeincrächtigung und

27 Biswcilcn auch als Grundrechutatbestand bezcichnet.

is Lübbe-Wolff. Die Grundrechic als Eingriffsabwehrrechse، 1988, S. 26; Schwabe, Probleme der Grundrechesdogma11k, r977, S. 1526.

29 Jurass, in: ders./Pieroth. Grundgeser\%, 4. Aurh, 1997. An. 202 Rdn. 2

3o Vgl. BVerwG, Besclsl. vom 13.4. 1995 . NJW 1995, 26.4\$ (2649) 
Sclutz der Naturressourcen nicht sclten in gleichem Maßc dem Einzelnen wie der Gesameheir dienende Ziele. Für die zu bescimnende Reichweire des von dem Umweltgrundrecht ausgehenden Schutzes kommt es somit entscheidend darauf an, der Verfolgung um welt relevanter Nurzungsinteressen hinreichend Frciraum zu belassen, oline dadurch die von jedem Menschen benörigeen ökologischen Grundlagen dauerhaft unbrauchbar zu nachen oder sie zu zerstören. Die vom Schutzbereich des Grundrcchtes erfaßłten Umweltgüter wären deshalb nur dann als berührr anzusehen, sofern zumindest zu erwarten stünde, daßs sie dem Menschen in der von ihm benötigten Weise künftig in nicht melır ausreichondem Maße zur Verfügung stehen. Anders formuliert, eine Becroffenheit des Schulzbereichs würde immer erst vorliegen, wenn cine Umweltcinwirkung die nachhaltige Nutzbarkcit von Naturressourcen in Frage stcillre. Dies beträle bei einer anthropogenen Belastung erneuerbarer Umweltgüter - wie dem Wasser - den Fall, daB deren baldige Erholung bzw. Wicderherstellung aus sich selbst heraus nicht mehr möglich ist. Der Umgang mic nicht oder nur begrenzt regenerativen Umweltgütcrn - wie dem Boden - cangierte den Schurzbereich, sobald dabci das Maß des Unvermeidlichen bzw. der jeweils größtmöglichen Ressourcenschonung uberschritcen wird, ohne daß effektive Ausgleichsoder Ersarzmaßnahmen getroffen werden (können). Hieraus wird deutlich, daßs cin solches Grundrechr in seiner inhaltlichen Reichweite jedenfalls über den Schurz der absoluc notwendigen Lebensvoraussetzungen im Sinnc eines irgendwie zu gewährleistenden ökologischen Exisrcnzminimums für den Einzelnen wcir hinausginge. Des weireren bedarf der Kreis derer, die durch das Umwelrgrundrecht als Betroffene der staaclicherseits herbcigeführten Umweltbeeinträchrigungen anzuerkennen wären, einer hinreichenden Konkretisicrung. Denn es wäre sinnwidrig und zudem kaum praktikabel, wenn etwa ein in Bayern beheimaterer und sich ständig dort aufhaltender Mensch zum Schurz des für ihn fernab gelegenen Wattenmeeres cigene Rechte geltend machen dürfte. Um Wildwüchsen dieser Art einen Riegel vorzuschieben, müßte dic Trägerschaft für das Umweltgrundrecht in qualifizierter und zugleich individualisierecr Weise an sclutzwürdige Interessen von Einzelpersonen geknüpft werden können. Dishalb kann Gegenstand der Einführung eines Umweltgrundrechtes auch nur die Bewahrung oder Wiederherseellung der für das eigene Leben wichtigen natürlichen Grundlagen sein. Eine dahingehende Eingrenzung hätte sich in erster Linie an Kricerien wic der Art der Umweltbelastung. der räumlich ausgerichreten Zuordnung der Umweltbeeinträchtigung und/oder der besonderen Eingriffsintensitäc bzw. -qualizät im Vergleich zu anderen Menschen auszurichcen. Als ein insoweit gecigneter Anknüpfungspunkt könnte die räumlich ausgerichtete Zuordnung der Umweltbeeincrächtigung dienen, so daß beispiclsweise an einen Schutz der natürlichen Lebensgrundlagen des Menschen in der fïr ibn den Schwerpunkt der Lebensbeziebungen bildenden näberen Umgebung zu denken wäre. Die nähere Umgebung ließe sich durch Inhalcsbestimmungen entweder unmiccelbar von Verfassungs wegen oder mituelbar durch einen konstiturionell verankercen Regelungsvorbehalt nach einfachgesetzlicher Maßggabe inhalclich weiter präzisicren. So bestünde ecwa die Möglichkeis, den Kreis der dadurch besrimmbaren Grundrechtsinhaber an eine nacl) (einwohner)melderechclichen Maßstäben zu bemessende Mindesraufentlıaltsdauer (z.B. von einem Monar) vor Ort zu knüpfen. Dem jeweiligen Wohnorr käme auf diese Weise eine zentralc Rolle für die abschließend zu definierende nähere Umgebung zu. $\mathrm{Ob}$ man diese dann an Verwalrungsgrenzen (z. B. die des Landkreises, in dem sicla der Wohnort befindet, bzw. der kreisfreien Stadh in der der Einzelne melderechrlich wohnhaft ist) orientiert festlegte oder aber einen nach Kilometern fixierten "Betroffenheirsradius « um den Wolsnort herum determinierte, bliebe durchaus diskussionswürdig. 
Während sich im Schutzbereich schuezwürdige Belange des Grundrechtsträgers verkörpern, rcalisieren sich bei einem Eingrilf in den Schutzbereich hoheitlich artikuliene gegenläufige Inceressen der Allgemeinheit oder eines Dritten. "Nach dem heute herrschenden Verständnis ist unter Eingriff jede nicht unerhebliche Einwirkung des Staates in ein grundrechtliches Schutzgut gegen den Willen des Grundrechtsträgers zu verstehen. ${ }^{32}$

Aufgrund dessen werden neben obrigkeitlichen auch schlichthoheitliche, verwaltungsprivatrecheliche und erwerbswirtschafiliclye Handlungen von Holicirsträgern erfaßi; neben rechesförmlichen Akten auch Realakte; neben den von der Stantsgewalt ausgehenden finalen Einwirkungen auf das Schuczgut auch ungewolle Wirkungen und objektiv verursachre (Neben-)Folgen; neben unmittelbaren auch mittelbar verursachte Becinträchtigungen des grundrechtlichen Schutzgutes. ${ }^{3} \mathrm{Jm}$ Ergebnis isı der solchermaßen expandierte moderne Eingriflstatbestand damit auf ein sehr weites Spektrum staatlicher Ingerenzen anwendbar.

Dieser ausgedehnte Eingriffsbegriff erlangt hier nicht zuletzt deshalb Bedeutung, wcil Beeinträchtigungen und daraus entstehende Nachteile für die natürlichen Lebensgrundlagen of mals eine immediate Konsequenz nicht hoheitlichen, sondern privaten Handelns sind. Man denke etwa an Verhaltensweisen der Betreiber industrieller Anlagen, der Autofahrer, der Verbraucher oder der Transporteure giftiger und sonstiger Stoffe. Wird jedoch berücksichtigh, daß vielen der für die Umwelr bedeutsamen nachteiligen Einwirkungen hoheitliche Gestattungen zugrundeliegen, stellen sich die dadurch mittelbar (mir)verursachten Schädigungen an Schutzgütern Dritter als (staatliche) Gundrechtscingriffc dar ${ }^{34}$ Aber auch wenn gestatrungsbcdürfriges umwelrschädliches Verhalten rechtswidrigerwcise ohne entsprechende behördliche Zulassung erfolgte, griffen die Mechanismen des Abwehrrechtes ein. In diesem Falle geschähe der unzulässige Umwelteingriff durch ein pflichtwidriges Nichteinschreiten der Behörden, der einen Anspruch des Einzclnen auf behördliche Abhilfe begnindcte. Entsprechendes würde dort gelten, wo niche gestatrungsbedürftiges, ökologisch schädliches Verhalten - erwa als Folge des Betriebes nicht genehmigungsbedürftiger Anlagen - abgewehrt werden soll.

Insofern bleibt zu konstatieren, daß dem Einzelnen gegen privat iniciierce Umweltbecinträchtigungen durchaus ein direkter Grundrechrsschutz zustehcn kann. Dieser sich dann freilich gegen den Träger öffentlicher Gewalt richtende Anspruch bewirkte einen Austausch des primären Konfliktgegners. Zu dem eigentlich nur zivilrechrlichen Abwehranspruch gegen den die Belastung unmitcelbar verursachenden Privaten geselle sich ein grundrechrlich legitimierter Anspruch gegen den Staat als im Vorleld der Beeincrächtigung verantwortlichen Mitakteur.

Grundrechte finden zwar wegen ihres ausschließlich gegen Hoheitscräger gerichrcen Defensiv-Charakters nach absolut herrschender Ansicht im Verhältnis von Privaten zueinander keine unmitrelbare Anwendung. "In Anbetracht des zuvor Ausgeführten

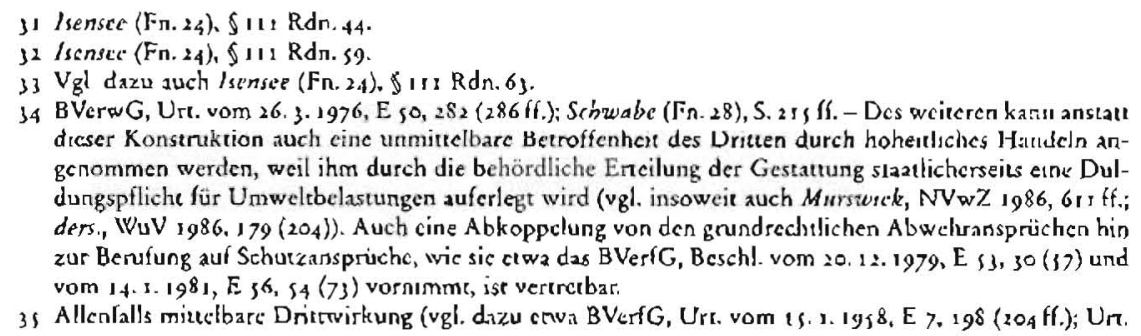


erscheint die Dritwirkungsproblematik für cin in das GG einzuführendes Umweltgrundrecht jedoch eher als "Scheinproblem «; zumindest aber ist sie kein - wie immer wieder behaupter - ernschaft die Wirkung eines Umweltgrundrechtes minderndes Hindernis.

Für die Qualifikation eines konkreten hoheitlichen Verhaltens als Eingriff bleiben im übrigen die allgemein für alle Grundrechte gleichermaßen geltenden Maßstäbc zu beachten. Danach stellen bloße Grundrechtsbelästigungen dem Einzclnen ohne weiteres zumutbare Bagatcllbeeinträchtigungen dar, die schon yon vornherein nicht als Grundrechiseingriffe gelcen. ${ }^{36}$ Umwelcbelastungen unrerhalb der Schwclle zur Gefährdung der nachhaltigen Nutzungsfäligkeit von Naturressourcen wcrden sogar nicht einmal den Schutzbereich eines Umweltgrundrechtes (oben II. 2.b.) tangieren können. Allerdings wird diesc Schwclle nicht immer leicht auszumachen sein. Dennoch gibt es insoweit einige durchaus brauchbare Indikatoren, wic beispielsweisc die Wichrigkeit des Schutzgutes, dessen Qualität und Empfindlichkeit, dic Intensitât der diesem drohenden Gefahr sowie in gewissem Umfange auclı die soziale Adäquanz.

Insgesamt reduziert sich somit die Zahl der vom Einzelnen wehrlos hinzunchmenden hoheidlichen bzw. hoheidlich mitverursachten Eingriffe in die Umwch becrächclich. Allcrdings sind auch durch cin Umweltgrundrecht negative Summacionseffekte - das sind die für sich genommen vielfach unbedeutenden Einzelbelascungen, die in Summe betrachcet zu erheblicken Umwelcschädigungen führen - schwer erfaßbar. Zur Abwehr von jenseits der deutschen Staatsgrenzen herrührenden Immissionen wird sich ein solches Grundrecht ebenfalls nur äußerst begrenzt eignen. Das Abwehrbegetyren des Grundrechtsinhabers könnte sich im Hinblick daraúf allenfalls gegen die bundesdeuischen Verantworcuingsträger richten. Von ihnen dürfre er indes lediglich verlangen, daß sie in den emittierenden Staaten mit geeigneten politisclien Mitreln darauf hinwirkten, damit auf deutsches Territorium übergreifende umweltschädigende Handlungsweisen unterblieben. Die Festlegung des dazu geeigneten Mittels bliebe aber den politisch verantwortlich Handelnden überlassen.

\section{d. Rechtfertigung des Eingriffs}

Der Grundrechtseingriff bedeutet nicht notwendig einen Grundrechtsverstoß. Er ist glcichwohl absolur unzulässig, sobald mit ihm der grundrechrliche Wesensgehalt berührt wird (Art. 19 Abs. 2 GG). Das betrifft bei einem zu statuierenden Umweltgrundrecht jedenfalls solche Unwelteingriffe, die - abstrakt betrachtet - zu einem unersetzbaren Verlust des Bestandes an den für ein (nach physiologischen Kriterien zu beurteilendes) gesundes menschliches Leben unbedingt norwendigen Naturgütern führen. Diese Naturgürer sind unantastbar.

Die Rechtfertigung des Eingriffs hängt ansonsten von der Struktur des betroffenen Grundrechts ab. Für die Ausgestaltung des Umweltgrundrechts ist deshalb von Relcvanz, ob es ohne Eingriffsvorbehalt bleibr oder mit einem solchen versehen wird.

Ein vorbchaldlos gewährleistetes Grundreche wärc eingriffsresistent ${ }^{37}$, so daß jeder Erlaß einfachgesetzlicher Eingriffsnormen bzw. von zu Eingriffen crmächcigenden einfachgesetzlichen Vorschriften zu unterbleiben hätte. Das Umweltgrundrecht unterläge diesenfalls nur den Begrenzungen, die ihm durch den cigenen Schutzbereich

vom Is. 1. 1958, E 7, 230 (233 6); Beschl, vom 23, 4. 1986, E73. 261 (269); Dïrig, in: Marna/Dïrig/Hcrzog,

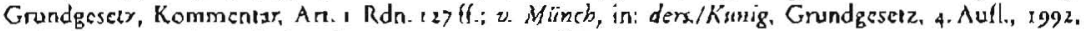

Vorb. Ar. 1-19 Rdn. 31 f.: Sparck, JuS 1981. 237 (213ff.))

36 So zurreffend: Kloepfor (Fn. 18). S. 20 f.: Pierotb/Schlink. Grundrechic, 19. Aufl., 1999, Rdn. 48.

i) Vgl. isensec (Fn. 24), 1ur Rdn. 69 . 
("grundrechrsimmanente Schranken «) sowie durch andere, ebenfalls verfassungsmäBig geschürztc Rechrsgütcr geserzr werden (»ver\{assungsiminanente Schranken $\propto$ ). ${ }^{3}$

Demgegenüber wäre das mit einem Geserzesvorbehalt ausgcstattere Umrvelıgrundrecht für Eingriffe in den Schutzbercich offen. Für diese sind die allgemein geltenden formellen wie materiellen Zulässigkeitskautelen maßgeblich. So müssen finale Eingriffe in formeller Hinsicht jedenfalls dem Geseczesvorbehalt genügen. Grundrechrsbeschränkende Geserze unterliegen dem Verbot der bloßen Einzelfallrcgelung und dem Zitiergcbor (Arr. 19 Abs. I GG). Materiell-rechtlich bleibr jeder Eingriff sters am Übermaßverbor zu messen. Insoweir hat zu geicen: Je wichriger das becroffene Umweltgut ais Lebensgrundlage des Menschen ist und je weniger dic potentielle Schädigung reversibel ist, desto geringer darf die noch zu rechtertigende Eingriffsintensität sein. Wenn es um Umweltgüter geht, ohne die menschliches Leben auf Dauer nicht oder nicht ohne eine Häufung schwerwiegender Krankheiten möglich ist, reicht daher schon eine sehr geringe Wahrscheinlichkeit der Zerstörung dieses Umweltgutes aus, um subjektive Abwehransprüche auszulösen.

Allerdings erzeugte ein durch privatc Dritte vermittelrer scaallicher Eingriff (z.B. die Erteilung einer Genchmigung) in die eingriffsoffen ausgestahteren, grundrechtlich geschützten narürlichen Lebensgrundlagen eine komplizierte Grundrechtsgemengelage. Hier wäre nämlich der dem staatichen Verhalten zurechenbare mitrelbare Eingriffsakt seinem Wesen nach zugleich ein Tcil der Grundrechtsverwirkdichung des privaten Dritten. Deshalb käme es in solchen Fällen regelmäßig zu Kollisionen zwischen dem subjektiven Recht des durch dic Umweltcinwirkungen in "seinem* Umweltgrundrecht Betroffenen und den durch die Art. 2 Abs, I, 12 Abs. I und I 4 Abs. 1 GG gewährleisteten Grundrechren des zivilen Umweltbceinurächtigers. Dies veranschauliche exemplarisch die behördlich zu treffende Entscheidung übcr die Zulassung einer großvolumigen Grundwasserentnabme zu gewerblichen Zwecken, welche die Austrocknung eines u. a. der Naherholung Dritter dienenden Feuchtgebieccs zur Folge haben kann. Der Entnehmende wird durch die Versagung oder Beschränkung einer die Grundwasserförderung zulassenden wasserrechtichen Erlaubnis oder Bewilligung $(\$ \$ 7,8 \mathrm{WHG})$ ebenso in einer grundrechtlich geschuitzten Position berührt (z.B. Berufsfreiheit i.S.d. Art. i 2 Abs. I GG, Recht am eingerichteten und ausgeübeen Gewcrbebetrieb i.S.d. Art. I 4 Abs. I Satz , GG) wie umgekehrt die durch die Auswirkungen der gescamecen Förderung negariv in sihren * Naturressourcen Betroffenen. Das hier hervortretende Konflikıpotencial zwischen höchst unterschiedlichen Grundrechten ließe sich jedoch einzelfallbezogen im Wege einer Rechtsgüterabwägung bereinigen. In diesem Rahmen müßten die konfligierenden verfassungsrechtlichen Positionen gegeneinander abgewogen und zum schonendsten Ausgleich gebracht werden. Angesichts dieses Konfliktlösungsmechanismus dürften die hier zweifelsfrei zutage tretenden Schwierigkeiten indes kaum cin ausreichondes Argument für die Ablehnung der Einführung eines (mir Eingriffsvorbehalt versehenen) Umweltgrundrechtes in das GG darstellen.

Soweir der Eingriff rechtswidrig ist, hat der davon betroffene Grundrechtsträger einen negatorischen Rechtsanspruch gegen den ihn beeinträchtigenden Hoheitsträger auf Uncerlassung oder auf Beseitigung. Gegcbenenfalls besteht ein Anspruch auf Ersaczleistung oder auf Folgenbeseitigung." Hälc der Eingriff in die naturlichen Lebensgrundlagen dagegen den verfassungsrechelichen Anforderungen stand, muß der Inhaber des Umweitgnundrechtes diesen dulden.

38 Allgemein dizu Hesse (Fn. 4), Rdn. 108 ff.

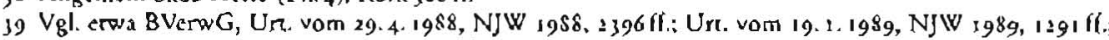
Heinizen, Verw Arch 81 (1990), s32 (s3s f.); Laubinger, Venvarch 80 (1989). 261 (292f.). 
Grundrechre beinhalten für den Einzelnen neben Abwehrrechten gegen Beeinträchugungen seitens der öffenclichen Hand auch Scluizpflichten für den Staat. Dicsc resultieren aus einer objektiv-rechtlichen Wercentscheidung der Verfassung für die von ihr umrissene und für alle Lebensbereiche geltende Rechtsordnung. Als solchermaßen verankcrte Rechtsprinzipien bilden die Grundrechte verbindliche Handlungsmaßsräbe für Legislative, Exekutive und Judikative. ${ }^{\circ 0}$ Sie erzeugen Richtlinicn und Impulse für die Staarsgewalt in Form von posiziven Handlungspflichten. ${ }^{+t}$ Inhaltlich erstrecken sich diese Verpflichoungen darauf, alles zu tun, um Grundrechte zu verwirklichen. ${ }^{42}$ Auch im Falle der Einführung eines Umwelrgrundreches in das GG hätre der Staat sich somit aktiv schützend und fördernd vor die vom Grundrechtsschucz erfaßicn nacürlichen Lebensgrundlagen zu stellen."3 Allerdings besceht nach Ansicht des Bundesverfassungsgerichesw ein Auspruch des Einzelnen auf hoheitliches Tátigwerden nur bei evidenten Verletzungen der in den Grundrechren verkörperten Wertentscheidungen. Da solche Verletzungen nach Verursachung, Intensicät und Wahrscheinlichkeit der drohenden Beeinträchrigung von Umwelrgütern für die staatliche Gewalt aber selten so eindeucig abschätzbar sind, dürften allein aus der staatlichen Schurzplicht abgeleirete Ansprüche des becroffenen Grundrechtsinhabers wohl die Ausnahme bilden. Im Hinblick auf ihren materiellen Wert weisen diese ansonsten nur objektiv-rechtlichen Schutzphichten in Zielsetzung und Strukrur deurliche Parallclen zu den Staatszielbestimmungen auf."s

\section{Umweltgrundrecht als Leistungsrecht}

Das Umweltgrundrecht würde zwangsläufig auch einen Iciscungsrechtlichen Einschlag haben. Dies gilc insbesondere hinsichtlich der Einstehenspflichc des Staates für dic nachhalrige Nutzbarkeit der narürlichen Ressourcen. Um diese dauerhaft zu gewährleisten, können aktive Vorkehrungen seitens der öffentlichen Hand erforàerlich sein. ${ }^{46}$

Voraussetzung dafür isc allerdings zunaclist, daß der Staat in der Lage wäre, über die nowwendigen Umweltgüter zu verfügen. Er müßte sie entweder selbst bereicstellen oder zumindest für ihre Bereirstellung durch private Dritte sorgen. Gegenwärtig sind zwar viele Umweltelemente weirgehend "freie* oder Privaten gehörende Güter. Dennoch werden die àazu zählenden natürlichen Lebensgrund̆lagen deswegen nichr vollends der staatlichen Verfügungsgewalt enczogen. Ungcachtec der Nichtinhaberschaft der Naturressourcen fungierı der Staat nämlich - entsprechend seinem aus Arr. $20 a$ GG resulciercnden Schuszaufurag - als Treuhänder für alle, auf den Schurz der natürlichen Lebensgrunalagen durch die ölfentliche. Hand angewiesenen Personen. Soweit ein im Privateigentum stehendes Umweltgur betroffen ist, wird die Verfügungsgewalt darüber indes durch die Grundreclute Dritcer, ctwa durch die Eigentumsgarancie des Art. ${ }_{4}$ GG, begrenzr.

40 Vgl. 8VcrfG, Bcschl. vom 8.8.1978, E 49, 89 (141); Beschl, vom 20. 12.1979, E s3. 30 (37): Beschl. vom 1.4.1.1981, E ,6, 54 (73); Besihl. vom 14.9.1983, NJW 1983, 2931 (2912); BGH, Urt. vom 10.12.1987. NIW $1088,478(481)$.

41 ข. Wiarch (Fn. 35). Vorb. Art. $1-19$ Rdn. 22.

42 Hesse, EuGRZ 1978, $\{17(41\})$

4) Oscenbisbl, in: Birburger Gesprache. Jahrbuch 198 3, 19\$3, 5 , (12): Karpen $(F n, 9)$, S. 14 .

$\$ 4$ Beschl. vom 34.9.1983, NJW [983. 2931 f.

is Hoppe/Beckmann, Umwclirach!, 1989. $\$ 4$ Rdn. 57

${ }_{4} 6 \mathrm{Vgl}$. dazu Sendler, DÖV $1978, s 81,(s 89)$. 
Immer wieder erhobene Bedenken, der Staat stoße im Falle einer dem Einzelnen gegenüber obliegenden Einstehenspflicht für die Nutzbarkeic der natürlichen Ressourcen leicht an die Grenze seiner finanziellen Leistungsfähigkeit, klingen vordergründig einleuchtend, greifen bei näherer Betrachicung aber zu kurz: Vielfach werden sich die entstehenden Kosten bereirs unter Anwendung des Verursacherprinzips auf den Verursacher überwälzen lassen. Insoweit bleiben die von der offentlichen Hand beansprucheen Anscrengungen im Ergebnis kostenneucral. ${ }^{47}$ Nur für die Fälle, in denen notwendigerweise das Gemeinlastprinzip zur Anwendung kommt, erliäl der Hinweis auf unkalkulierbare finanzielle Folgen Substanz. Das betrifft in erster Linie •öffendichea Altlasten (z.B. ehemalige militärische Liegenschaften oder Abfalldeponien) und Altlasten Privater, bei denen kein leiscungsfähiger Verantworticher (mehr) greifbar ist, sowie Naturgüter (z.B. Wälder, Moorlandschaften), deren Erhaltung den Einsatz öffentlicher Mirtel (z.B. Finanzicrungshilfen) erfordert. Doch lassen sich auch diese Bedenken crhcblich relacivieren. So will die Verfassung mit ihren Positivierungen dic Staatsmacht jedenfalls nicht zu unmöglichen Lcistungen verpflichten. Das Bundesverfassungsgericht ${ }^{4^{8}}$ betont in seinem Numerus-clausus-Urceila, daß das, was der Grundrechrsinhaber an Leistungen (bzw. Teilhabe) beanspruchen könne, unter dem »Vorbehale des Möglichen im Sinne dessen, was der Einzelne vernünfrigerweise von der Gesellschaft beanspruchen kann , steht. Forderungen bis an den Rand der Zahlungs- und Handlungsunfähigkeir des Staates sind demnach ausgeschlossen. Mit Blick auf die Einstehenspflicht der öffentlichen Hand bedeutete dies, hier könnte vom Inhaber des Umweltgrundrechtes unabhängig von konkreten Gefährdungen seiner Schutzgüter Leben, Gesundheit und Eigenoum - freilich nur nach Maßgabe des soeben Ausgeführten - auch die Beseitigung bestehender oder dic Abwendung drohender Beeinträchrigungen der natürlichen Lebensgrundlagen beansprucht werden.

Ob sich aus dem Umweltgnundreche überdies ein Anspruch gegen den Staat auf Einräumung subjcktiver Landschaltsbetretungsrechte herleiten ließe, läßt sich pauschal nicht beantworten. Denn der Schutz der natürlichen Lebensgrundlagen kann es gelegendich geradezu erfordern, daß ihre Zugänglichmachung verhinderr wird.

Daß eine solche Leistungskomponente zur Vollziehbarkeir u.U. erst noch der cinfachgesetzlichen Ausformung, gegebenenfalls zusätzlich der gerichtlichen Konkretisicrung bedüfte, hindert die Einführung des Umweltgrundrechces entgegen anders lautender Behauptungen nicht. Auch der aus Art. 1 Abs. y und 2 Abs. 2 sowie Art. 2 Abs. I GG i.V.m. dem Sozialscaatsprinzip abgelcicete Anspruch auf Sicherung des Existenzminimums durch Fürsorge (wSozialhilfe«) ${ }^{44}$ oder der aus Art.7 Abs. ${ }_{4}$ GG herausfilcrierte Anspruch privacer Ersarzschulen auf staacliche Förderung ${ }^{\circ 0}$ bedarf und erfährt dcrartige Präzisierungen.

\section{s. Umweltgrundrecht als Einrichcungsgarantie}

Grundrechue können schließlich auch auf die Gewährleistung bestimmter Einrichcungen ziclen." Dicse Einrichrungsgarantien wirken vornehmlich konservierend und

\footnotetext{
47 Murswiek, in: Isensee/Kirchhof (Hrsg.), Handbuch des Suatsrechrs, Bd. V, 1992, 112 Rdn. 24 I.

$48 \mathrm{Urt}$, vom 18.7.1972, E 33, 303 (333).

49 State vieler Dürig (Fn. 19), Ar. I Rdn. 43 ; Art. 2 Abs. 11 Rdn, 26 l.

so BVerfG. Ur. vom 8.4.1987, E 75, $40\left(\sigma_{2}\right.$ ff.).

32 Mauny, Deutsches Stzalsrecht, 24. Aufl., 1982, S. 110.
} 
sichern so gesehen das einmal Erreichte. ${ }^{22}$ Der Grundrechtekatalog des GG schürzt auf diese Weise beispielsweise die Ehe und Familie (Art. 6 Abs. I), den Religionsunterriche (Arr. 7 Abs.3) oder das Eigcntum und das Erbrechr (Art. 4 Abs. 1). Syscematisch hier anzusiedeln wäre z.B. dic dem Umweltgrundrechc zu enrnehmende "Kernbestandsgarantie für das geltende Umweltrecht (oben I. 2.).

\section{Fazit}

Zusammenfassend blcibc somit zu resümieren, daß die Einführung eincs Umweltgrundreches in das GG richt nur zu einer spürbaren Stärkung des rechtlichen Umweltschutzes führen würde, sondern überdies kompatibel mir der bestehenden Grundrechtssystemacik wäre. Auch die damit untrennbar einhergehende Prakrikabilität eines Umweltgrundrechtes ließe sich durchaus sicherscellen. Maßgeblich bliebe insoweit allein die inhaltliche Ausgestaltung, die dieses Grundrecht erhielte. Freilich vermag cin Umwelrgrundrecht nicht alle umweltschutzrechtlichen Probleme zu lösen. Es kann insbesondere nichr das sich im wesentlichen subkonstiturionell vollziehende Umweltschurzreche erseczen, es jedoch komplementieren. Auch ist es dem Inhaber dieses Grundrechtes darnit nicht möglich, den von jenscits der deurschen Grenzen oder aus bloßen Summationseffekten herrührenden Umweltbelasstungen wirksam entgegenzutreren. Dennoch würde ein solches Grundrecht dem bislang eher "zahnlosen matericllen Umweltverfassungsrechi des GG immerhin ein gebrauchstaugliches Gebiß verleihen.

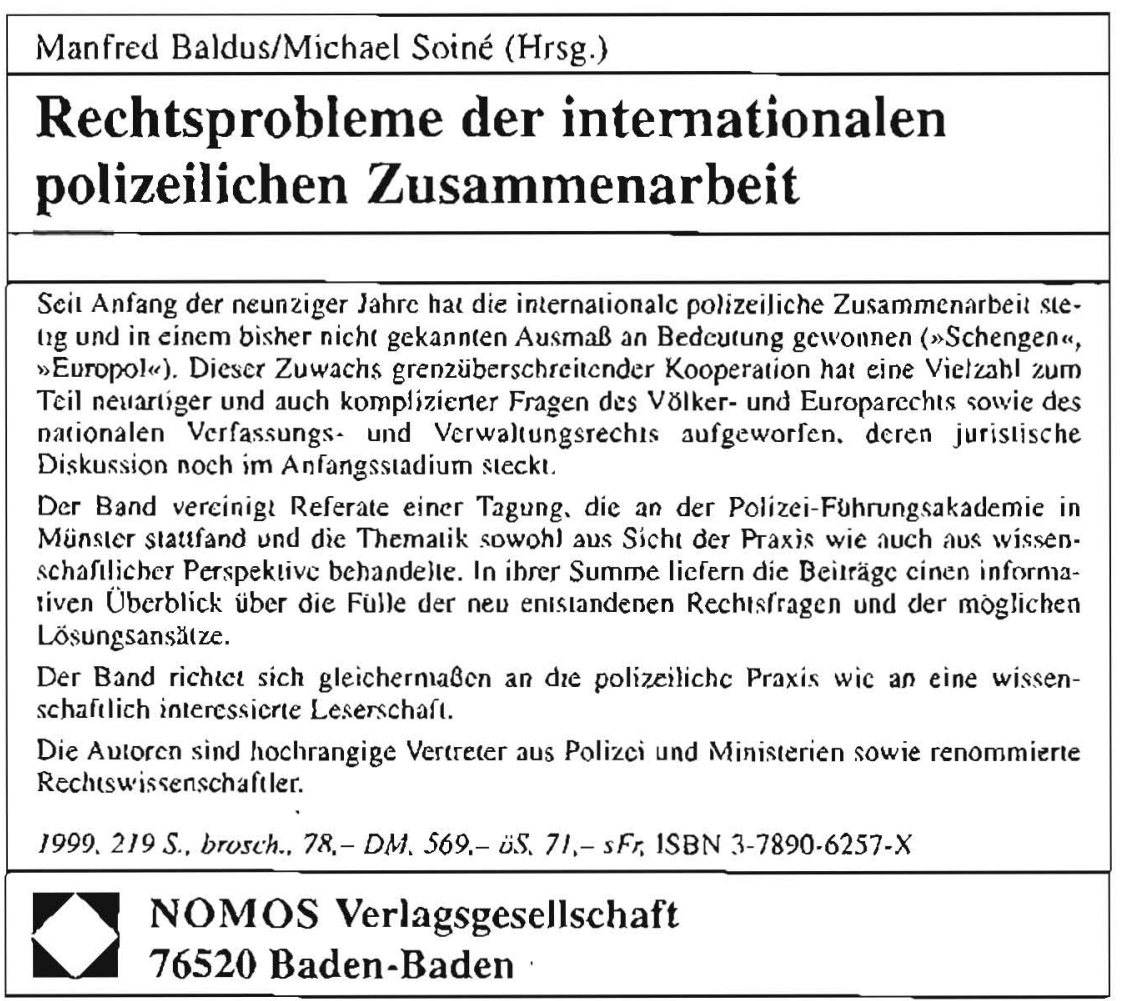

32 Wrpfelder, ZRP $1986,140(145)$. 